\title{
Life-Sized Projector-Based Dioramas
}

\author{
Kok-Lim Low Greg Welch Anselmo Lastra Henry Fuchs
}

\author{
University of North Carolina at Chapel Hill \\ Department of Computer Science, Campus Box 3175 \\ Chapel Hill, NC 27599-3175, U.S.A. \\ $+1-919-962-1700$ \\ \{lowk, welch, lastra, fuchs\}@cs.unc.edu
}

\begin{abstract}
We introduce an idea and some preliminary results for a new projector-based approach to re-creating real and imagined sites. Our goal is to achieve re-creations that are both visually and spatially realistic, providing a small number of relatively unencumbered users with a strong sense of immersion as they jointly walk around the virtual site.

Rather than using head-mounted or general-purpose projectorbased displays, our idea builds on previous projector-based work on spatially-augmented reality and shader lamps. Using simple white building blocks we construct a static physical model that approximates the size, shape, and spatial arrangement of the site. We then project dynamic imagery onto the blocks, transforming the lifeless physical model into a visually faithful reproduction of the actual site. Some advantages of this approach include wide field-of-view imagery, real walking around the site, reduced sensitivity to tracking errors, reduced sensitivity to system latency, auto-stereoscopic vision, the natural addition of augmented virtuality and the provision of haptics.

In addition to describing the major challenges to (and limitations of) this vision, in this paper we describe some short-term solutions and practical methods, and we present some proof-ofconcept results.
\end{abstract}

\section{Categories and Subject Descriptors}

I.3.7 [Computer Graphics]: Three-Dimensional Graphics and Realism - virtual reality; I.3.6 Methodology and Techniques interaction techniques; I.3.8 Applications.

\section{Keywords}

Spatially-Augmented Reality, Virtual Reality, Augmented Virtuality, Virtual Environment, Shader Lamp, Diorama, Multiprojector Display System, Immersive Visualization, User Interface.

Permission to make digital or hard copies of all or part of this work for personal or classroom use is granted without fee provided that copies are not made or distributed for profit or commercial advantage and that copies bear this notice and the full citation on the first page. To copy otherwise, or republish, to post on servers or to redistribute to lists, requires prior specific permission and/or a fee.

VRST'01, November 15-17, 2001, Banff, Alberta, Canada.

Copyright 2001 ACM 1-58113-385-5/01/0011 ..\$5.00.

\section{INTRODUCTION}

Many of us have fond memories of unusual places we have visited at one time or another, perhaps as adults, perhaps as children. The combination of sights, sounds, smells, and spatial experiences often leaves a lasting mental imprint. Museums and theme parks often try to evoke similar feelings by physically recreating sites that are far away or perhaps lost with time. For example, Walt Disney World's Epcot Center in Orlando, Florida (USA) includes a "World Showcase" area of the park where
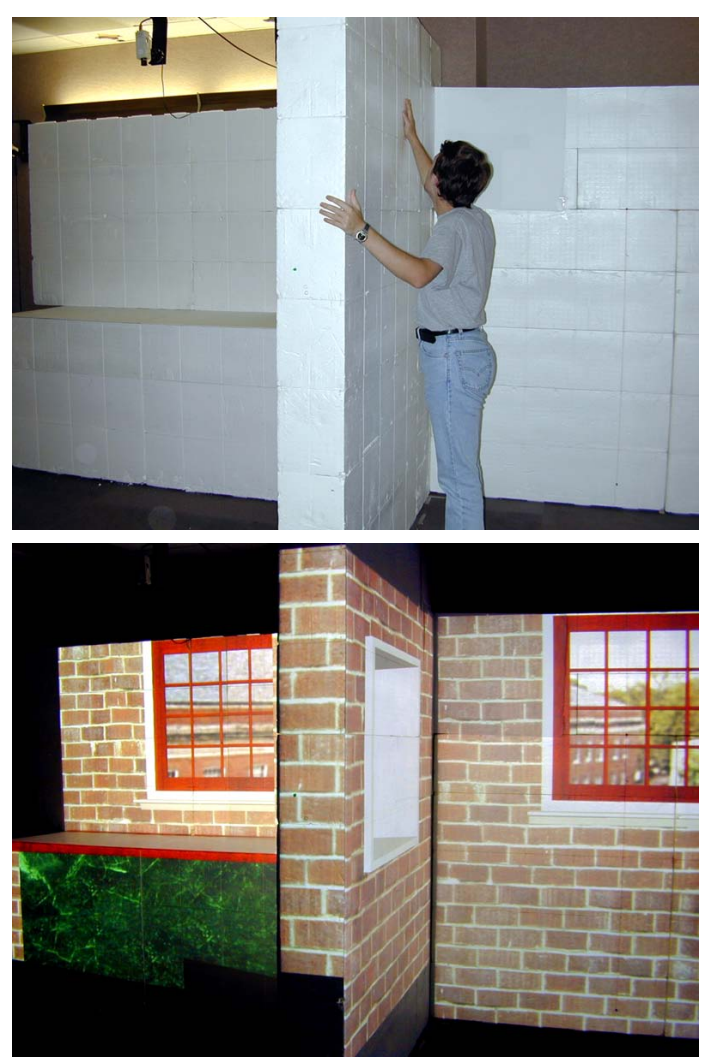

Figure 1. We use Styrofoam blocks to construct a geometrically simplified replica of the scene. The Styrofoam blocks are used as display surfaces on which view-dependent imagery of the scene is projected. Most of the geometric complexities in the scene, such as the window frames, windowsills, the pass-through in the middle wall, and the molding around it, are left out in the simplified replica. (This figure is reproduced in color on page 000.) 
small portions of cities from around the world are re-created with movie set-like façades, and workers who are natives of the actual city. At the other end of the real-virtual spectrum are purely virtual examples such as the London Traveler [24], which uses virtual reality to allow tourists to rehearse a visit to London before actually arriving.

What we seek is something in-between such completely real and virtual examples, a spatially realistic and visually virtual hybrid reconstruction. We want to enjoy the richness, flexibility, and dynamic nature of computer graphics, while preserving some of the physical and mental activity associated with walking around or exploring a real site. We want to provide users with a realistic sense of presence in both synthetic and real places. While we are only working in our laboratory at the moment, we envision a day when museums dedicate a modest area to life-sized projectorbased dioramas of famous places such as Monticello ${ }^{\dagger}$, U.S. President Thomas Jefferson's home in Charlottesville, Virginia.

\subsection{Life-Sized Projector-Based Dioramas}

Head-mounted displays (HMDs) have been commonly used for immersive visualization of virtual environments. With headtracking, HMDs can provide real-time view-dependent stereo views of the virtual world. Unfortunately, views through HMDs are usually very narrow, and can detract from a person's ability to navigate through an environment effectively, and decrease his sense of presence in the virtual environment [3]. Further, HMDs typically occlude most (or all) of any peripheral view of the real world and other nearby people, which makes the user reluctant to move freely.

Projector-based visualization systems such as the CAVETM [6] surround the user with large screens that are fixed in the environment (not on the user). The screens are illuminated with perspectively-correct stereo imagery from light projectors placed behind the screens. While this approach improves the field of view, it restricts the area (typically $3 \times 3 \times 3$ cubic meters) the viewer can physically walk around when exploring virtual environments. Placing the screens far away to enclose enough walking space may not be a good solution for various reasons. For example, when the viewer is at the center of a CAVE looking at a mid-sized virtual object very near to him, the far-away screens have to be very tall in order to allow the projection of the complete image of the object.

In this paper, we introduce a new projector-based approach for re-constructions of real and synthetic sites. Our approach was inspired by Michael Naimark's "Displacements" exhibit at the San Francisco Museum of Modern Art in 1984 [18]. Naimark used a rotating movie camera to film a static living room, replete with furniture and (still) people. The room and furniture were then painted white, and the captured imagery was projected back onto the walls using a rotating projector that was precisely registered with the original camera.

We introduce an interactive paradigm that extends and generalizes Naimark's ideas. Using simple white building blocks we construct a static physical model that approximates the size, shape, and spatial arrangement of a remote (synthetic) site. We

\footnotetext{
${ }^{\dagger}$ http://www.monticello.org
}

then project dynamic imagery onto the blocks, transforming the lifeless physical model into a visually-faithful reproduction of the actual site. Besides increasing the spatial realism of the virtual environment, our approach has many other advantages over the application of traditional head-mounted or CAVETM-like displays.

We have conducted some preliminary experiments using a synthetic scene, and the results are encouraging. While we are making progress toward our goal to re-create real places, we do not yet have a complete system. As such, throughout the paper, we present a mix of demonstrated results from new methods, and plausible ideas for future systems. We do our best to distinguish between the two lest the reader be led to believe that we have implemented something that we have not.

In the following section, we present the basic idea behind our approach, and we discuss its advantages and limitations. In Section 3, we look at the issues of implementing a system based on our new approach, and the challenges we face. Then, we demonstrate some results from our preliminary experiments before we conclude the paper.

\section{NEW APPROACH}

Of course, one way to realistically re-create a place is to actually build an exact replica of it, complete with all surface details, colors and reflectance/photometric properties. Except for permanent dedicated sites (e.g., Disney's "World Showcase") this is typically impractical. Even if practical, the result will generally be a static model of the site.

Instead our approach was closely based on the idea of shader lamps [19], which extends and generalizes Naimark's ideas. The concept of shader lamps is based on the observation that when we illuminate a physical object with a white light, its surface reflects particular wavelengths of light that we perceive as color. Alternatively, certain physical attributes can be effectively incorporated into the light source to achieve a perceptually equivalent effect using colored light on a white object. With shader lamps only the geometric structure of the scene needs to be replicated, while digital light projectors fill in the surface details and colors. Still, the geometric structures of real places are usually complex and creating exact geometric replica can be a daunting task. Instead our approach is to use a geometrically simplified replica of the scene as the projection surface. The simplified surface is easier to plan and build. In addition to filling in surface details and colors, we use the projectors to render view-dependent imagery where needed to compensate for the geometric differences between the virtual scene and the simplified physical model (display surface).

We believe the extension of shader lamps is appropriate for our goal. For example, primary structures of building interiors and mid-sized architectural objects (walls, columns, cupboards, tables, etc.), can usually be approximated with simple components (boxes, cylinders, etc.). As seen in Figure 1, we are using construction-grade Styrofoam blocks (from Reddi-Form, Inc. [23]) in our preliminary experiment. The main architectural features that match the simplified physical model retain 3D autostereoscopic views, while any other (more subtle) details must be presented by projecting view-dependent images. 
In general, different degrees of approximation of the scene geometry produce a spectrum of display surfaces that range from single flat screens (a CAVE ${ }^{\mathrm{TM}}$ ) to display surfaces that exactly match the scene geometry. For our goal, we want to simplify the display surfaces as much as possible but still preserve the empty space where the user may walk, bend over, or put his arm through, for instance. However, in Section 2.1, we will see that there are many advantages to having the display surfaces as similar as possible to the scene geometry.

\subsection{Advantages}

Although the initial motivation is to provide more spatial realism to the user, our approach has many other advantages that are also essential for achieving our goal of re-creating real places. Some of these advantages are listed below.

\subsubsection{Wide FOV and Peripheral Vision}

Human vision has an effective field of view (FOV) that spans approximately 200 degrees horizontally by 150 degrees vertically. Many commercially available HMDs have relatively narrow fields of view, ranging from roughly 30 to 70 degrees diagonally. A narrow FOV has been shown (in real environments) to degrade human performance on navigation, manipulation, spatial awareness, and visual search tasks, and to disrupt our eye- and head-movement coordination and our perception of size and space [8][2][3]. Peripheral vision is known to be well-suited to maintaining self-orientation during locomotion [14], and there is evidence to suggest that a narrow field of view detracts from a person's ability to navigate through an environment effectively, and decreases his sense of presence in the virtual environment.

One of the main motivations of the CAVE system and our approach is to provide visualization with a very wide FOV. This is done by using large projection screens that are fixed in the environment at some distance from the user. Doing this also has other advantages over HMDs-the user no longer needs to wear heavy gear on his head, and fast head rotations are less likely to produce serious swimming effects [6].

\subsubsection{Real Walking}

With our approach, the physical arrangement of the display surfaces allows the user to really walk around in the virtual environment. Real walking gives stronger sense of presence than walking-in-place and virtual flying [27], but at the expense of larger physical space.

With systems such as CAVE, the virtual environment that the user explores can be larger than the enclosed physical space. In such a situation however, users can only navigate using less natural methods such as walking-in-place or pressed-button virtual flying.

\subsubsection{Reduced Sensitivity to Tracking Error}

Like general-purpose projector-based setups such as the CAVETM $^{\mathrm{TM}}$ where the display surface is fixed in the environment rather than to the user (as in a HMD), our approach is relatively insensitive to error in estimates of the head orientation. See [6] for a complete explanation. However unlike general-purpose projector-based systems, our approach is also relatively insensitive to error in estimates of the head position.

The diagram in Figure 2 illustrates the geometry of a general projector-based setup from a top view. This will be used to illustrate error in a horizontal plane. The vertical error would be similarly derived. The point $V$ represents some point on the graphics model, i.e. a point on the virtual surface. If the tracker reports the viewpoint is at $E_{1}$, the subsequently rendered image of point $V$ will appear on the physical display surface at point $D_{1}$. However if the eye point is actually located at point $E_{2}$, then the image of point $V$ should have been rendered on the display surface at point $D_{2}$. As a result, the user will experience an angular viewing error of $\theta_{E}$.

Note that the shape of the display surface is irrelevant; because the point $D_{2}$ is not being rendered, it does not matter where it should appear (anywhere on the line $V E_{2}$ ), the user will still experience the same error, $\theta_{E}$. Similarly the absolute position of the eye point is not the critical factor, what matters is the relative position of the actual eye point with respect to the rendered point $D_{1}$.

In fact, the angular error $\theta_{E}$ depends only on three parameters: $V E_{2}$, the distance from the virtual scene point to the user's eye point; $V D_{1}$, the distance from the virtual scene point to the physical display surface; and $\theta_{V}$, the angle between the estimated and actual eye points. Given those three parameters and some trigonometric manipulation, the angular viewing error $\theta_{E}$ can be computed as

$$
\theta_{E}=\operatorname{asin}\left(\frac{\sin \left(\theta_{V}\right)\left|V D_{1}\right|}{\sqrt{\left|V D_{1}\right|^{2}+\left|V E_{2}\right|^{2}+2\left|V D_{1}\right|\left|V E_{2}\right| \cos \left(\theta_{V}\right)}}\right)
$$

The two surface plots in Figure 3 illustrate the angular viewing error $\theta_{E}$ throughout a space approximately the size of a CAVE ${ }^{\mathrm{TM}}$, for display surface distances $V D_{1}$ from a virtual point at the far corner of the plot $(0,0)$ of 10 and $50 \mathrm{~cm}$. To cover the space, $\theta_{V}$ is varied from 0 to approximately 90 degrees, and $V E_{2}$ is varied between 0 and 2 meters.

Note from the above equation and plots that the angular error (a) increases as the angle $\theta_{V}$ between the estimated and actual eye points increases, (b) decreases as the distance $V E_{2}$ from the virtual scene point to the actual view point increases, and (c)

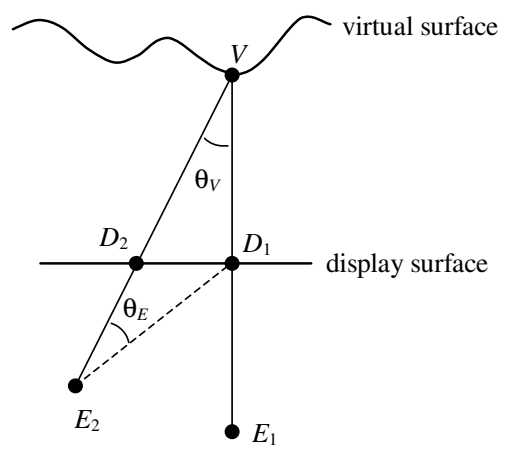

Figure 2 

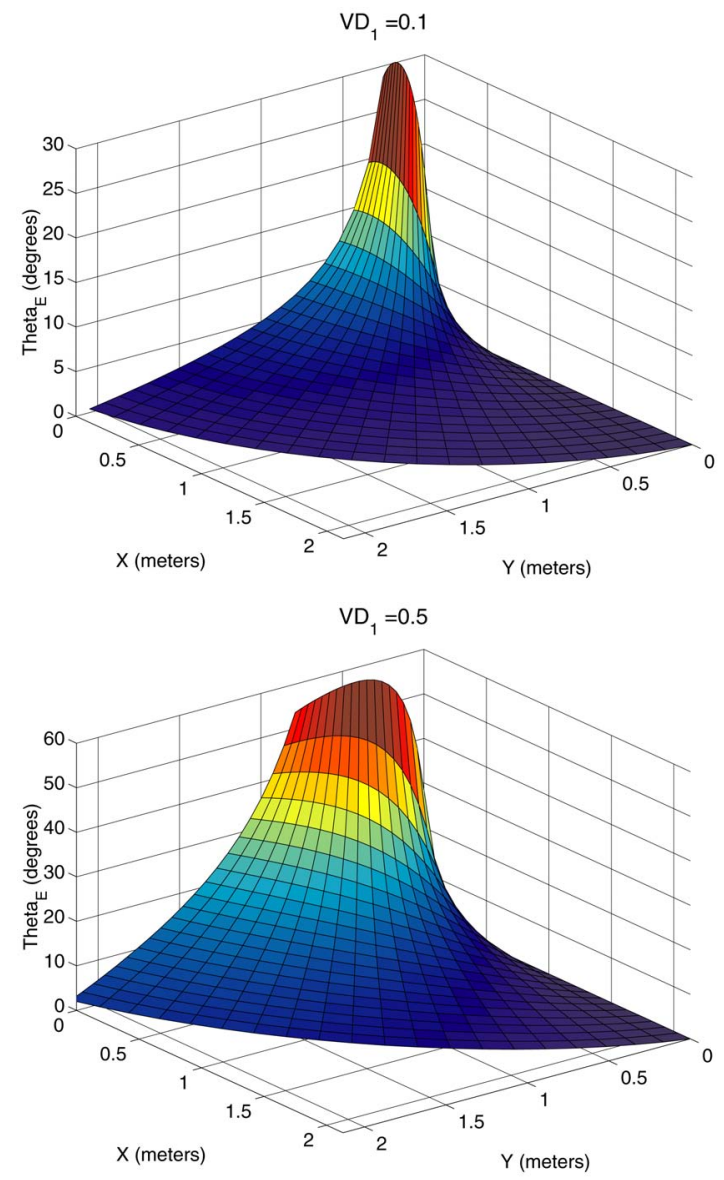

Figure 3

increases as the distance $V D_{1}$ between the real and virtual surface increases. The first and second cases are likely familiar to most people who have experience with conventional computer graphics and perspective projection. However the last case is less frequently recognized or discussed in conventional computer graphics, most likely because the display surface and the virtual objects are typically very different. The important thing to note is that even if the display surface is not precisely located and shaped like the corresponding virtual portion of the scene, if it is relatively close, and if the viewer does not get too close to the display surface, the angular error can be relatively small.

Note that if $V D_{1}=0$, i.e. if the physical display surface exactly matches the corresponding virtual objects, then the angular error $\theta_{E}$ is 0 no matter what the values of $V E_{2}$ and $\theta_{V}$. In other words, in that special case you would not even need a tracking systemyou would simply "paint" the display surface with the proper colors.

\subsubsection{Reduced Sensitivity to Latency}

The sensitivity to view position error with HMDs and generalpurpose projector-based displays magnifies the effect of unavoidable latencies in the graphics pipeline. The problem is that the scene ends up being rendered from the wrong viewpoint: from where the user was, rather than where he actually is. Such latency-induced viewing errors can break a user's sense of presence or immersion, and can even cause motion sickness.

In contrast, because our scene-specific approach enjoys reduced sensitivity to tracker error, it consequently also enjoys reduced sensitivity to system latencies. People typically translate their heads relatively slowly, and even fast head rotations cause only a relatively small change in the eye positions.

The effect of reduced sensitivity to latency can be readily observed in our prototype system, where system latencies are quite noticeable for the outdoor views through the window (where $V D_{1}$ is quite large), but are much less noticeable at the windowsills and the molding around the pass-through (where $V D_{1}$ is relatively small).

\subsubsection{Natural Auto-Stereoscopic Vision}

When the geometry of the display surfaces matches that of the virtual environment exactly, the images of the scene projected onto the display surfaces are naturally 3D stereoscopic, without the use of any special 3D stereo projection. After all, the user is viewing the actual 3D surfaces. When the set of display surfaces is a simplified model of the scene, only parts of the scene that match the display surfaces will retain auto-stereoscopic views, and other parts must be reproduced using special 3D stereo projection. In our preliminary experiment, we have many large walls in the scene that match the simplified display surfaces. Even without the use of stereo projection, the virtual scene still looks three-dimensional because most of the surfaces are very close to the actual desired geometry.

\subsubsection{High Spatial and Geometric Fidelity}

In traditional graphics rendering, spatial and geometric properties of an object are presented in images. The resolutions of these images can limit the accuracy with which the geometric properties are presented. However, using our approach, parts of the scene model that match the display surfaces' geometry can be presented with very high spatial and geometric fidelity [19]after all, aside from color, the user is viewing a real physical object.

\subsubsection{Eye Accommodation}

As mentioned by Cruz-Neira [6], eye accommodation (eye focus) provides depth cues in the real world. In typical computer graphics, HMD VR and CAVE, eye accommodation has not been incorporated in visualization, and everything in the virtual environments is in focus. With our approach and the approach of the shader lamps, the user can now selectively focus his eyes on any part of the scene, thus providing the user a very natural way to visualize the virtual environment.

\subsubsection{Augmented Virtuality}

Because the physical arrangement of the display surfaces is spatially similar to that geometry of the scene, it is relatively easy to add real objects to the virtual environment. For example, if a set of display surfaces approximates a virtual desk, then we can put a vase on the desk by placing a real vase on the set of display surfaces that approximates the desk. Additional projectors can then be used to properly light up the added real object to simulate the effect of the virtual lights in the virtual environments. 


\subsubsection{Haptics}

Clearly, if the set of display surfaces is sufficiently close to the scene surfaces, we can use it to provide the user what Fred Brooks has called static haptics [5][13]. In an experiment setup, Brooks et al. have used low-fidelity, low-cost and easily configurable physical structures to construct very approximate physical model for a kitchen. The user wears a HMD and moves in the physical model, able to touch it physically, and also see a very detailed image of the kitchen. The effect of actually feeling the surfaces is a very powerful component in achieving a sense of presence. Their experience has been that the user's visual sense overrides small errors in the positioning and lack of fine details of the physical model.

\subsection{Drawbacks and Limitations}

Probably the most significant limitation of our idea is the scenespecific nature of the implementation. Clearly the approach is not applicable for general-purpose visualization, where a CAVETM like display or a HMD is likely to make more sense. As indicated by the title, the idea is primarily useful (we believe) for creating life-sized projector-based dioramas of real or imagined sites.

\subsubsection{Physical Aspects}

Given a decision to use our approach to re-create a particular site, clearly the next most significant concerns are the physical aspects related to the need for a physical display surface model, and for the physical space to house projectors, cameras, and of course the physical model. We believe that the requirement for a physical model is more onerous now than it will be in the future. As described in Section 3.3 we have some tools to help us now, and we envision more powerful computer-aided-assembly tools in the future. With respect to the physical space requirements, we are now working in a research laboratory so we cannot simulate a place larger than it. However, we envision a warehouse-sized structure in which we can re-create most indoor scenes. It is also useful to note that we do not always need to allocate physical space for everything in the scene-we only need to allocate physical space for those parts of the scene that we want the user to move into. For example, in Figure 1, no physical space is allocated for the outdoor scene seen through the windows.

\subsubsection{Scene Content}

We enjoy most of the advantages outlined in Section 2.1 only if we keep the physical surfaces similar to the scene surfaces. This means that dynamic scenes with large objects that move a great deal or deform significantly are not good candidates for our approach. However, small objects that move near large surfaces (for example, an insect crawling on a wall) should not pose much difficulty to our approach. Moreover, of relevance to our goal, most museums exhibit static artifacts, which visitors are not allowed to touch.

\subsubsection{Projector Considerations}

In general, our approach does not always allow back-projection to be possible. For example, in Figure 1, both sides of the middle wall are to be illuminated and there is virtually no space in between them to put in projectors. Although the back-projection approach has a higher space requirement, it has the advantage that users will not occlude light from projectors. Instead we envision a "sea of projectors" arranged throughout the ceiling of the reconstruction space. While it might be an issue now, we believe that projector size and cost will not be a significant consideration in the future. As noted in [28] the size and cost of projectors continues to decrease at a rapid pace. Today you can purchase a projector that weighs less than five pounds, has $1,000+$ lumens of brightness, for under $\$ 5,000$. New, reflective micro displays based on standard CMOS technology offer the hope of smaller, smarter, and less expensive digital projectors.

\section{IMPLEMENTATION}

We have implemented a very preliminary prototype to demonstrate the idea of our new approach, but we do not yet have a complete system. As such, here, we do our best to distinguish between what we have implemented and what we have not.

\subsection{Overview}

A system based on the new approach will have the following main components:

- Scene modeling. The scene model can be synthetic or of a real place.

- Display surface modeling. A model of the display surfaces must be computed to fit the surfaces in the scene model.

- Physical display surface construction. A physical setup has to be constructed in accordance to the computed display surface model.

- Projector placement and calibration. The projectors are placed at the appropriate places with respect to the physical model, and are then calibrated to find their geometric relations with the physical model.

- Tracking. Each user's head must be tracked in order to generate perspectively-correct images.

- Rendering. This is responsible for generating correct imagery on the display surfaces as viewed from the user's position. It must also take care of the overlapping projections from different projectors, and produce images that blend seamlessly at the overlap regions.

Each of these components is discussed in greater detail in the following subsections, in which we present the main issues, the challenges, our solutions and/or other possible ways to address the challenges.

\subsection{Scene Modeling}

The main challenge here is when we want to re-create real places. To model a real place, we can use a range scanner to sample the geometry of the scene, and a camera to sample the photometric properties of the scene. Some of the issues encountered are the management of the enormous amount of data acquired, the registration of the photographs with the range data, and the registration of the range data from different views [15][17]. If view-dependent surface reflection is to be simulated, then many more photographs of the real scene have to be taken, 
and a light-field approach can be used to render the viewdependent images [29].

Currently, the most practical interactive rendering method for VR is still the traditional polygon-based z-buffer approach. Therefore, surface reconstruction from range data to generate a polygonal model [7] is necessary. Mesh simplification [11] is then applied to reduce the number of polygons by eliminating redundant geometric information. During rendering, photographs of the real scene can then be textured onto the simplified mesh.

While we currently only demonstrate our ideas with a synthetic scene, we have collected and are in the process of preparing very high-quality image-based models of the Monticello library. We acquired the models using a 3rdTech [1] laser scanner during a multi-day trip to Monticello with our UVA collaborator David Luebke.

\subsection{Display Surface Modeling and Construction}

The need to model and build non-trivial physical display surfaces is a challenge not seen in other projector-based approaches. One of our main objectives is that the physical display surfaces should be easy to setup. This has led us to consider Lego ${ }^{\text {TM}}$-like blocks from a set of predefined simple shapes. An open question is what is the best general set of predefined shapes?

With a selected set of predefined shapes, we would like to have a method to automatically compute an arrangement of the blocks to best fit the surfaces of the scene model. This is a very difficult problem, and we do not see any similar problems in the literature.

In our prototype, we use $0.25 \times 1.2 \times 0.3$ cubic meters white stackable Styrofoam blocks to set up the physical display surfaces. To create a display surface model in the computer, we have implemented a program to allow us to manually design an arrangement of the blocks to fit the scene model. This model is then used as the blueprint to roughly construct the physical setup. Later when the projectors are calibrated, we project wireframe images of the display surface model onto the physical blocks to help us fine-tune and correct the physical setup.

In the future we envision using the projectors and the analytical models of the display surface to guide users in the assembly of the physical model. Given an empty space with calibrated projectors, an automated program could render the outline of a block on the floor, waiting for the assembly worker to confirm placement of the block. Similar steps are applied to the remaining blocks, including stacked blocks.

\subsection{Projector Placement and Calibration}

\subsubsection{Projector Placement}

After the display-surface model has been created, we need to decide where we want to place the projectors. We may also want to know the minimum number we need to cover all the display surfaces. This problem is similar to that of the camera placement in image-based modeling [9][25], but it is made more difficult by the fact that, besides their positions, we also want to compute the projectors' orientations.

A method to compute a good set of projectors' positions and orientations would want to take into consideration the following:
- physical and environmental constraints on the projector mounting,

- maximizing the area of coverage of each projector,

- minimizing overlap area,

- maintaining a certain image resolution on display surfaces,

- minimizing possible occlusion by the user,

- $\quad$ minimizing inter-reflections among display surfaces, and

- $\quad$ minimizing the number of projectors.

In order to minimize inter-reflections of light, it is best to avoid projecting light on a large surface at a very oblique angle.

We have not yet automated the projector placement. This is an open area of research. Currently we mount the projectors (using flexible brackets) in places that we believe accommodate the above considerations, then typically try a few variations before settling on the final pose.

\subsubsection{Projector Calibration}

After the projectors are positioned and oriented appropriately with respect to the physical display surfaces, we adjust their focusing distances to get the best focus on the display surfaces. The next step is to calibrate the projectors to find their geometric relations with the scene model and the display surface model.

To calibrate a projector, we need to find a set of paircorrespondences. Each pair-correspondence consists of the coordinates of a $3 \mathrm{D}$ point in space, and its corresponding pixel coordinates on the projector's image plane. With sufficient paircorrespondences (at least six, and no four 3D points are coplanar), we can solve for the projection parameters using linear least-square methods or nonlinear optimization approaches [10]. To find a pair correspondence, we project a known 2D point from the projector, which emerges from the projector as a ray. Then, we move a small display surface to intersect the ray, and use a tracker to measure the 3D position of the intersection. These 3D points are then transformed from the tracker coordinate system into the coordinate system of the scene model, using a known transformation. This set of pair-correspondences is used to solve for the projector's projection parameters with respect to the scene's coordinate system.

The above manual approach to finding pair-correspondences can be tedious and error-prone at times. In the future, automatic projector calibration using cameras such as in [22] might be implemented, possibly as part of an automated projectorplacement algorithm.

\subsection{Tracking}

Since the user is allowed to freely walk around within a potentially large area, we need a wide-area tracking device to track his head position. If the user's limbs are also allowed to interact with the environment, then they need to be tracked too. The required spatial resolution and accuracy depend largely on the largest $V D_{1}$ in the model (see Figure 2). The larger the distances between the desired model and the physical display surfaces, the more important the spatial fidelity of each tracked view. 
We use two 3rdTech's [1] HiBall trackers in our experiment. These trackers can cover a very wide area and have sufficiently high precision and low latency. One tracker is used to track the user's head and another is tracking the position and orientation of a virtual spray can. Each position returned by the trackers is transformed into the coordinate system of the scene model.

\subsection{Rendering}

\subsubsection{Generating Perspectively-Correct Images}

For each projector, we use a two-pass rendering approach to generate the correct images for the user. In the first pass, the scene model is rendered normally from the position of the user's eye. The resulting image is read back from the framebuffer, to be used in the next pass. In the second pass, the display surface model is rendered from the projector's viewpoint, with the display surfaces texture-mapped with the image created in the first pass. The texture map is mapped onto the display surfaces using projective texture mapping, projected from the position of the user's eye [21]. The image read-back from the framebuffer can be a performance bottleneck because transferring the highresolution image can be very demanding on the memory bandwidth of many rendering systems.

\subsubsection{Display Surface Partitioning}

To provide a panoramic view to the user, we have to project images on all the display surfaces around the user. Consider the situation in Figure 4: two walls are illuminated by a projector and the user is in between the two walls. During the first rendering pass, we need to render an image of the scene from the viewer's position. However, there is no way we can set up a view frustum that can generate a complete image of the two walls. In order to generate complete images of the two walls, we can set up two different view frusta, each one looking at a wall, and render two images. During the second rendering pass, the display surfaces that approximate each wall are texture mapped with the corresponding image created in the first pass. This requires that the display surfaces be separated into groups so that each group can be texture mapped with a different image. In our prototype, we have partitioned the display surfaces manually.

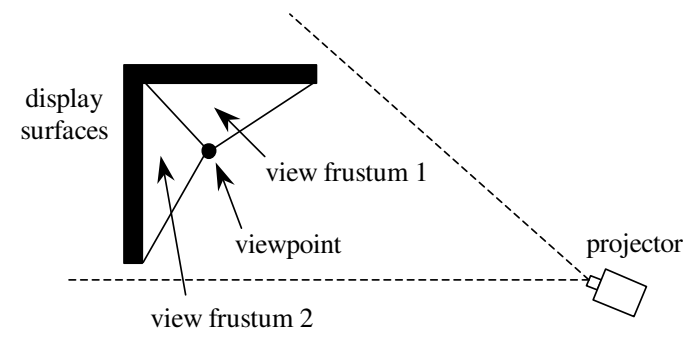

Figure 4

\subsubsection{Seamless Blending of Projections}

Regions of the display surface that are illuminated by multiple projectors appear brighter, making the overlap regions very noticeable to the user. To make the overlap regions appear seamless, we can use alpha blending techniques to reduce the intensity of each projector's projection in the overlap region [22]. However, our display surfaces can have concave regions that can cause the overlap regions, as seen from a projector, to be non- contiguous. Traditional intensity roll-off method cannot handle this case very well, but there is a better method proposed in [19].

Another problem of merging images from multiple projectors is the lack of color equivalence between neighboring projectors [16]. Majumder et al. use hardware color look-up tables to correct for the color mismatch between projectors.

We have not implemented any correction for overlap regions and color differences in our prototype system.

\subsection{Other Issues and Challenges}

\subsubsection{Inter-Reflections}

From our preliminary experiments, we have observed that interreflections of light from the projectors can be a serious problem. They degrade the quality of the projected images by reducing their contrast and color saturation. We do not yet have a good solution to this problem, but we believe the following guidelines are helpful:

- $\quad$ use less reflective (more diffuse) display surface material,

- avoid overly oblique projections on large surfaces.

In the future we envision an automated approach that takes the latter (and other heuristics) into account.

\subsubsection{Shadows}

One major problem of using front-projection approach for our purpose is that the projectors may be occluded by the user. Our experience with our prototype has been that the shadows can be quite annoying. We can place the projectors higher and closer to the display surfaces, but this is not a good solution because the projectors will be at very oblique angles to the display surfaces. A possible solution is to have at least a "backup" projector for every projector, and a projector and its "backups" will illuminate the same surface from different locations but not at the same time. Video cameras and trackers are used to determine which projectors the user has occluded and the "backups" will be called in to fill in the shadows.

\subsubsection{Multiple Viewers}

If the physical display surfaces match the virtual scene geometry, then multiple viewers should be able to explore the environment simultaneously, and everyone will always see the correct images, without the need for tracking. (See "Reduced Sensitivity to Tracking Error" in Section 2.1.3.) However this extreme requirement for the physical model is unrealistic or unreasonable to expect for most situations we can imagine. This means that the need to support multiple simultaneous independent head-tracked views remains an issue. We are not alone in this respect-

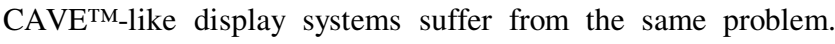
Unhappily there does not appear to be a practical solution readily available to support more than a few (two, perhaps three) simultaneous head-tracked views.

The problem includes difficulties in tracking, rendering, and display. While tracking and rendering pose difficult challenges, the most difficult problem related to multiple viewers is the actual display. Like conventional general-purpose projectorbased display systems, we are affected by both practical limitations on projector technology and the fundamental physics 


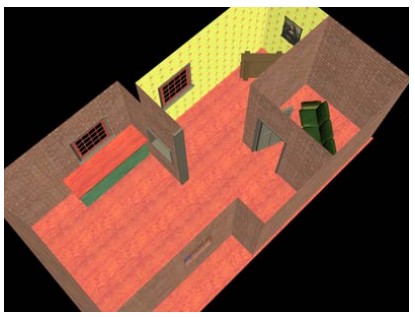

(a)

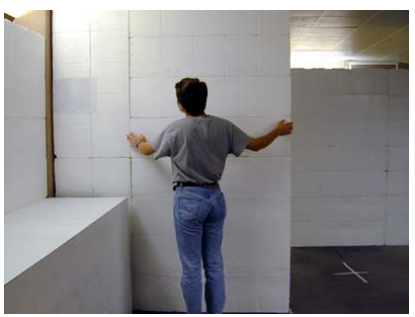

(c)

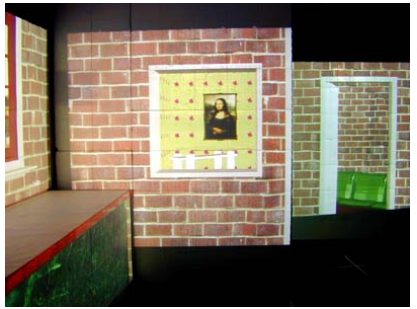

(e)

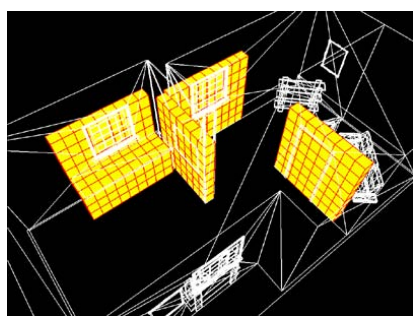

(b)

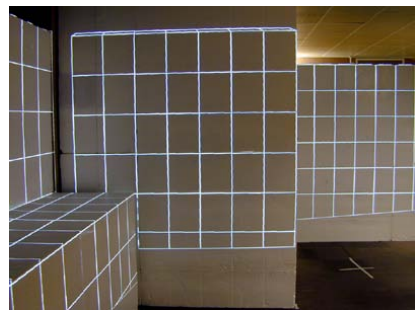

(d)

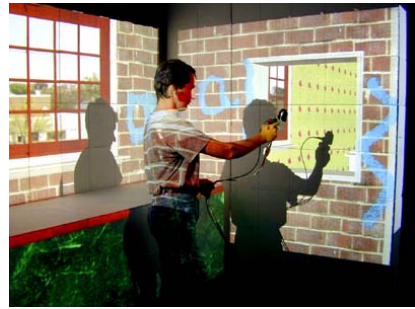

(f)
Figure 5. The prototype. (a) The synthetic scene model, (b) the display surface model, (c) a rough physical arrangement of the display surface model, (d) verification of physical model using wireframes projected from the projectors, (e) the final result with perspectively-correct imagery generated in real-time, (f) a user is virtually spray-painting on the virtual walls. (This figure is reproduced in color on page 000.)

of light. Traditional methods for projecting multiple images "simultaneously" on the same surface include time-division and phase multiplexing. Time-division multiplexing is limited by the speed at which one can change projector images, and by the decreasing amount of light corresponding to shorter projection intervals as you add more views. Phase multiplexing (polarized imagery) is limited by the fundamental ways you can polarize light, the ways that projectors internally process the light (sometimes independently polarizing it), and the effects of polarization on the quality of the final imagery. The ability to deliver independent imagery channels to each individual's eyes is arguably a significant advantage that head-mounted displays have over projector-based displays. We have some ideas to address these shortcomings, but are not prepared to elaborate at this point.

\section{PRELIMINARY RESULTS}

We have implemented a prototype to demonstrate our ideas. In this prototype, we simulate only a part of a synthetic model of a room (Figure 5), because of the limited space in our research laboratory-which is about $8.5 \times 4.5 \times 2.5$ cubic meters. We created the physical display surfaces using construction Styrofoam blocks (Figure 5(c)). Six projectors were used, each with an image resolution of $1024 \times 768$ pixels. Two HiBall trackers were used-one to track the user's head and another to track a virtual spray can. We used a 32-processor, 8-pipe SGI Onyx2 Infinite Reality2 ("Reality Monster") to generate all of the images for the projectors, in real time. Due to the limited number of projectors and the relatively slow two-pass rendering, we have not included stereo projections in our system.

Our experience is that the results were compelling, especially when looking through the virtual pass-through in the protruding wall, while walking from one side of the wall to the side. Also compelling is to see the outside imagery move through the window, passing behind the mullions, while the window frame and surrounding walls and counters remain fixed in the room with the user. In Figure 5(f), we show a user doing virtual spraypainting on the walls and window frame. The purpose is to demonstrate the usefulness of being able to physically move around virtual objects-which gives the user a natural way to spatially interact with the scene.

We are in the process of building-up the model to include the walls on the right of the camera in Figure 5(e), to provide a further panoramic (immersive) effect.

\section{CONCLUSION}

We are encouraged by the results of our first prototype. The ability to walk around corners of the environment is, we believe, unique. As a next step, we intend to try a model acquired at Monticello. This will present a much greater challenge because the furniture in the rooms contain many more curved surfaces, and are thus not a good match to our building blocks. We believe that the transition to the complex model will require research on more optimal block shapes and sizes, as well as automated fitting of the physical model to the virtual.

Since we drive the projectors, we can potentially exert very precise control over the lighting in the room. Furthermore, we already track the participant's eyes, so we may be able to enhance the experience by removing user-induced shadows using backup projectors as described earlier, and lighting the participant's bodies while keeping the projected light out of their eyes.

A more practical enhancement that we plan for the next version of the system is the transition to a cluster of PCs. This will enable us to increase the number of projectors and perhaps scale the system performance. With these, we also hope to be able to include stereo projections into our future prototype.

\section{ACKNOWLEGEMENTS}

We wish to thank Ron Ardres and Reddi-Form, Inc. for their generous donation of Styrofoam building forms (blocks), as well as Herman Towles, Jim Mahaney, David Harrison, and John Thomas for their local technical assistance. Although not actually incorporated into our demonstrations yet, the Monticello laser data set mentioned throughout was collected in collaboration with Prof. Lars Nyland at UNC-Chapel Hill, and Prof. David Luebke at the University of Virginia. Adrian Ilie has implemented part of the system and helped in shooting some of the photographs that appear in this paper. 
Support for this research comes from NSF Cooperative Agreement no. ASC-8920219: "NSF Science and Technology Center for Computer Graphics and Scientific Visualization," and the supplemental grant "High-Fidelity Tele-Immersion for Advanced Surgical Training."

\section{REFERENCES}

[1] http://www.3rdtech.com

[2] Patricia L. Alfano and George F. Michel. Restricting the field of view: perceptual and performance effects. Perceptual and Motor Skills, 70(1):35-45, 1990.

[3] Kelvin W. Arthur. Effects of Field of View on Performance with Head-Mounted Displays. Ph.D. Dissertation, Department of Computer Science, UNC Chapel Hill, April 2000.

[4] Frederick P. Brooks, Jr. What's Real About Virtual Reality? IEEE Computer Graphics \& Applications, 19(6):16-27, November 1999.

[5] Frederick P. Brooks, Jr. Static Haptics. Personal communication, 1999.

[6] Carolina Cruz-Neira, Daniel J. Sandin, Thomas A. DeFanti. Surround-Screen Projection-Based Virtual Reality: The Design and Implementation of the CAVE. Proceedings of SIGGRAPH 93, July 1993.

[7] Brian Curless, Marc Levoy. A Volumetric Method for Building Complex Models from Range Images. Proceedings of SIGGRAPH 96, pp. 303-312, 1996.

[8] Hubert Dolezal. Living in a world transformed: perceptual and performatory adaptation to visual distortion. Academic Press, 1982.

[9] Shachar Fleishman, Daniel Cohen-Or, Dani Lischinski. Automatic Camera Placement for Image-Based Modeling. Proceedings of Pacific Graphics 99, 1999.

[10] Olivier Faugeras. Three-Dimensional Computer Vision. MIT Press, 1993.

[11] Michael Garland, Paul S. Heckbert. Surface Simplification using Quadric Error Metrics. Proceedings of SIGGRAPH 97, pp. 209-216, August 1997.

[12] Mark Hereld, Ivan R. Judson, Rick L. Stevens. Introduction to Building Projector-Based Tiled Display Systems. IEEE Computer Graphics and Applications, pp. 22-28, 2000.

[13] Brent Insko. Passive Haptics Significantly Enhances Virtual Environments. Ph.D. Dissertation, Department of Computer Science, UNC Chapel Hill, April 2001.

[14]H. W. Leibowitz. Recent advances in our understanding of peripheral vision and some implications. Proceedings of the 30th Annual Meeting of the Human Factors Society, pp. 605-607, 1986.

[15] Marc Levoy et al. The Digital Michelangelo Project: 3D Scanning of Large Statues. Proceedings of SIGGRAPH 2000, August 2000.
[16] Aditi Majumder, Zhu He, Herman Towles, Greg Welch. Achieving Color Uniformity Across Multi-Projector Displays. Proceedings of the IEEE Conference on Visualization 2000, 2000.

[17] David K. McAllister, Lars Nyland, Voicu Popescu, Anselmo Lastra, Chris McCue. Real-Time Rendering of Real World Environments. Proceedings of the 10th Eurographics Workshop on Rendering, 1999.

[18] M. Naimark. Displacements. An exhibit at the San Francisco Museum of Modern Art, San Francisco, CA (USA), 1984.

[19] Ramesh Raskar, Greg Welch, Kok-Lim Low, Deepak Bandyopadhyay. Shader Lamps: Animating Real Objects with Imaged-Based Illuminations. Proceedings of the 12th Eurographics Workshop on Rendering, June 2001.

[20] Ramesh Raskar, Greg Welch, Matt Cutts, Adam Lake, Lev Stesin, Henry Fuchs. The Office of the Future: A Unified Approach to Imaged-Based Modeling and Spatially Immersive Displays. Proceedings of SIGGRAPH 98, July 1998.

[21] Ramesh Raskar, Matt Cutts, Greg Welch, Wolfgang Stuerzlinger. Efficient Image Generation for Multiprojector and Multisurface Displays. Proceedings of the 9th Eurographics Workshop on Rendering, 1998.

[22] Raskar, Ramesh, Michael Brown, Ruigang Yang, Wei-Chao Chen, Greg Welch, Herman Towles, Brent Seales, Henry Fuchs. Mutli-Projector Displays Using Camera-Based Registration. Proceedings of the IEEE Conference on Visualization 99, pp.161-168, October 1999.

[23] http://www.reddiform.com/

[24] Anthony Steed, Emmanuel Frécon, Anneli Avatare, Duncan Pemberton and Gareth Smith. The London Travel Demonstrator. Proceedings of the ACM Symposium on Virtual Reality Software and Technology, pp. 50-57, December 1999.

[25] Wolfgang Stuerzlinger. Imaging all Visible Surfaces. Proceedings of Graphics Interface 99, 1999.

[26] Susuma Tachi, T. Maeda, R. Hirata, H. Hoshion. A Construction Method of Virtual Haptic Space. Proceedings of the 4th International Conference on Artificial Reality and Tele-Existence (ICAT'94), 1994.

[27] Martin Usoh, Kevin Arthur, Mary C. Whitton, Rui Bastos, Anthony Steed, Mel Slater, Frederick P. Brooks, Jr. Walking > Walking-in-Place > Flying, in Virtual Environments. Proceedings of SIGGRAPH 99, August 1999.

[28] Greg Welch, Henry Fuchs, Ramesh Raskar, Michael Brown, and Herman Towles. Projected Imagery In Your Office in the Future, IEEE Computer Graphics and Applications, July/August 2000 20(4): 62-67.

[29] Daniel N. Wood et al. Surface Light Fields for 3D Photography. Proceedings of SIGGRAPH 2000, pp.287-296, August 2000. 